\title{
Farewell to a pioneer
}

\section{Bernard Lovell, builder of Jodrell Bank's iconic radio telescope, has died.}

In northwest England, the ground rises on land that was owned in the fourteenth century by William Jauderell, an archer in the army of Edward, the Black Prince. The rise became known as Jodrell Bank.

By 1939 it had been colonized by the botany department of the nearby University of Manchester. In 1945, Bernard Lovell arrived at the site with various bits of left-over war-time equipment with which he intended to study cosmic rays - escaping the trams that caused electrical interference as they passed the Manchester physics department and founded the Jodrell Bank Observatory.

By mid-1957, Lovell had built using bits of left-over battleship - the
'Mark I' telescope, at the time the largest steerable-dish radio telescope in the world and the only device capable of tracking the launch of the Sputnik satellite. In 1966, it eavesdropped on Luna 9, the first spacecraft to land on and transmit from the Moon.

A host of astronomical observations followed too, including the first complete Einstein ring in 1998. Renamed the Lovell telescope, and now part of the MERLIN array, this landmark in both radio astronomy and the British countryside is testament to the inventiveness and determination of its creator.

Bernard Lovell died on 6 August 2012 at the age of 98 .

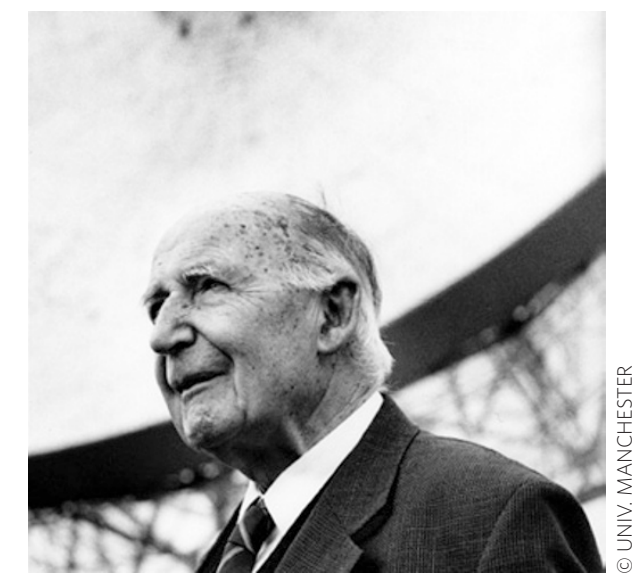

\section{...and farewell to the Pioneer anomaly}

\section{The unexpected deceleration of the Pioneer space probes has, at last, an explanation.}

Between 1958 and 1978, NASA's Pioneer programme saw the launch of a series of unmanned space probes into the Solar System. Pioneer 10, launched in March 1972, was the first mission to Jupiter and the first spacecraft to enter the asteroid belt beyond the orbit of Mars. Its twin, Pioneer 11, followed in April 1973, and used the gravity of Jupiter to set it on course for the first encounter with Saturn.

The missions were a great success Pioneer 11 even making the first foray through the plane of Saturn's rings, ahead of the Voyager probes that would follow from 1977 - and, decades later, the images returned by these 260 -kilogram craft are still stunning. But the Pioneers, true to their name, kept going. And going. Communication with Pioneer 11 was finally lost in 1995, and with Pioneer 10 only in 2003 when it had reached a distance of 80 astronomical units from Earth.

However, some years previously, when the spacecraft had travelled about 20 astronomical units, it was realized that both were thousands of kilometres closer to the inner Solar System than they should be, according to calculations. Both Pioneers seemed to be subject to some small deceleration that NASA hadn't reckoned on. In 1994, a serious investigation of this 'Pioneer anomaly' began - and, as a result of a frankly Herculean effort by a small group of scientists, the solution seems now to be found.

Of course, from the outset it was clear that there were several mundane possibilities to explore in seeking an explanation for the anomaly: gas leakage or uneven heat radiation from the identical spacecraft, for example, or simply measurement error. But the unexpected deceleration also raised other possibilities - for instance, that the laws of gravity might not be quite what we expect at such astronomical distances. Although observations of the outer planets, and of Neptune's moons, rule out a straightforward modification of gravity, a modification of inertia (involving a kind of large-scale Casimir effect) has been mooted.

For decades, the anomaly remained. The Pioneers were realized to be uniquely sensitive to the decelerating force, cruising smoothly over huge distances because they were spinstabilized: other craft, including the Voyagers, were not, and their regular firing of thrusters to set or maintain course overwhelmed any sensitivity they might have had to the tiny Pioneer anomaly. There is some hope that NASA's New Horizons mission, launched in 2006 and set to reach Pluto in 2015, might display a measurable anomalous deceleration, as it too is spin-stabilized for a chunk of its voyage; however, the design of the craft might result in some degree of unpredictable thermal-radiation pressure that would again overwhelm the effect.

With supporting data unlikely, then, to emerge from other sources, a group of scientists, including Slava Turyshev and Viktor Toth, set about a detailed study of the Pioneer craft and the data from them. That's no mean feat: data collected in the 1970s and 1980s isn't easily readable in the twenty-first century; what data hadn't been deleted resided on antiquated magnetic tapes and had to be 'upconverted' for use - "that took years", Turyshev has said.

Neither do CAD (computer-aided design) files exist for spacecraft launched 40 years ago. Instead, Turyshev and the team had to resort to blueprints and any other project documentation they could recover to build a finite-element thermal model of the Pioneer craft, laboriously plugging coordinates of the vertices of each surface into the modelling software. When all that was done, the greatest source of uncertainty in the thermal model turned out to be due to the paint that coated the Sun-facing surfaces of the crafts' radioisotope thermoelectric generators "three mils of zirconia $\left(\mathrm{ZrO}_{2}\right)$ in a sodium silicate binder", for which there is no literature on performance and likely degradation in the conditions on board the Pioneers.

Nevertheless, the outcome of almost two decades of effort from Turyshev et al. now published in Physical Review Letters $\mathbf{( 1 0 8 , 2 4 1 1 0 1 ; 2 0 1 2 ) ~ - ~ i s ~ a ~ d e t a i l e d ~ m o d e l ~}$ of the likely history of the spacecraft that suggests they've been subject to a thermal recoil force that tallies neatly with the 'anomalous' deceleration. Once again, the mundane possibilities have won out against exotic, new-physics explanations. But the determination and perseverance of the scientists who have resolved the Pioneer anomaly is heroic indeed. 